\title{
Sharp Convergence Rates for Nonlinear Product Formulas*
}

\author{
By Eric Schechter
}

\begin{abstract}
Nonlinear versions of the Lie-Trotter product formula $\exp [t(A+B)]=$ $\lim _{n \rightarrow x}[\exp ((t / n) A) \exp ((t / n) B)]^{n}$ and related formulas are given in this paper. The convergence rates are optimal. The results are applicable to some nonlinear partial differential equations.
\end{abstract}

0. Introduction. The Lie-Trotter product formula states that

$$
\left[\exp \left(\frac{t}{n} A\right) \exp \left(\frac{t}{n} B\right) \exp \left(\frac{t}{n} C\right)\right]^{n} \rightarrow e^{t(A+B+C)} \text { as } n \rightarrow \infty .
$$

(The exponent $n$ indicates iterated composition.) This is a special case of a more general principle: if $T(t)(t \geqslant 0)$ is a family of operators with $T(0)=I$, and if $T^{\prime}(0)$ exists in some appropriate sense, then

$$
T(t / n)^{n} \rightarrow \exp \left[t T^{\prime}(0)\right] \quad \text { as } n \rightarrow \infty .
$$

These product formulas have been studied in [3], [4], [13], [14], [16] and elsewhere. They are most familiar when $A, B, C, T^{\prime}(0)$ are square matrices. But $(0.1)-(0.2)$ and other such formulas are also valid for some nonlinear, discontinuous operators $A, B$, $C, T^{\prime}(0)$ in an arbitrary Banach space; hence the product formulas are applicable to some nonlinear partial differential equations. Such formulas also serve as prototypal models for some approximation schemes in numerical analysis [7], [9], [11], [12], [17].

How fast are the convergences in (0.1)-(0.2)? This depends at least in part on the regularity of the operators involved: we may get faster convergence if we assume more regularity. In the best possible case, when $A, B, C$ are continuous linear operators, we get the fastest possible convergence:

$$
\left[\exp \left(\frac{t}{n} A\right) \exp \left(\frac{t}{n} B\right) \exp \left(\frac{t}{n} C\right)\right]^{n}-e^{t(A+B+C)}=O\left(\frac{t^{2}}{n}\right)
$$

as $n \rightarrow \infty$, uniformly for bounded $t$. This is, again, a special case of a more general principle: If $D$ and $T(t)$ are continuous linear operators, and if

$$
\left\|\left(T(t)-e^{t D}\right) / t^{\beta+1}-Q\right\|=o(1) \quad \text { as } t \downarrow 0,
$$

Received September 6, 1983.

1980 Mathematics Subject Classification. Primary 65J15; Secondary 34G20, 47H20.

Key words and phrases. Accretive, alternating direction method, approximation scheme, composition, convergence rate, dissipative, evolution, exponential, fractional step method, resolvent, semigroup, split step method.

* Supported in part by a grant from the Vanderbilt University Research Council. 
for some nonzero remainder $Q$, then we have convergence at the rate

$$
T(t / n)^{n}-e^{t D}=O\left(t^{\beta+1} / n^{\beta}\right)
$$

and no faster. This will be proved in 5.9.

Thus the estimate in (0.3) cannot be improved. But we can obtain faster convergence by using more complicated formulas. For instance, for continuous linear operators,

$$
\begin{aligned}
{\left[\frac{1}{2} \exp \left(\frac{t}{n} A\right) \exp \left(\frac{t}{n} B\right) \exp \left(\frac{t}{n} C\right)+\frac{1}{2} \exp \left(\frac{t}{n} C\right) \exp \left(\frac{t}{n} B\right) \exp \left(\frac{t}{n} A\right)\right]^{2} } \\
-e^{t(A+B+c)}=O\left(t^{3} / n^{2}\right)
\end{aligned}
$$

and

$$
\begin{aligned}
\exp \left(\frac{t}{2 n} A\right)\left[\exp \left(\frac{t}{n} B\right) \exp \left(\frac{t}{n} A\right)\right]^{n-1} & \exp \left(\frac{t}{n} B\right) \exp \left(\frac{t}{2 n} A\right) \\
-e^{t(A+B)} & =O\left(t^{3} / n^{2}\right) .
\end{aligned}
$$

These formulas were discussed in a different form in [18]. Again, the rates are best possible.

Surprisingly, the optimal rates (0.3)-(0.5), which are achieved for continuous linear operators, are also achieved for a large class of discontinuous, nonlinear operators. That is the main result of this paper.

Our results are stated in terms of an abstract class of operators in a Banach space. The setting is more general than the usual setting of numerical analysis; still, it is specific enough so that we obtain sharp convergence rates. The setting is somewhat more specialized than the usual setting of nonlinear semigroup theory. Nevertheless, the setting is still general enough so that our results are applicable to several kinds of mildly nonlinear partial differential equations with smooth coefficients. For instance, they are applicable to parabolic equations; to hyperbolic equations, during the brief interval before shocks develop; and to dispersive equations, such as the Korteweg-de Vries equation. These diverse equations have in common the property that they preserve smoothness in the spatial variables. That is, if the solution $u(t)$ of one of these equations begins at an initial value in some appropriate Sobolev space $W^{m, p}(\Omega)$, then $u(t)$ will remain in that space for at least a while, and it is possible to give an upper estimate for $\|u(t)\|$. We shall take $m$ large, so that the solution has extra smoothness in the spatial variables; it follows that we can perform calculations with $u^{\prime}(t), u^{\prime \prime}(t)$, etc.

The theory developed here is entirely local in time. Hence it is applicable regardless of whether solutions exist globally. This is useful because the solutions of some differential equations blow up after finite time [1].

The class of operators considered in this paper is slightly more complicated and less general than the class of " $\nu$-generators" studied in [15], [16]. In Section 6 we shall show that the generators of the present paper are a subclass of the $\nu$-generators. A version of (0.1) was proved for $\nu$-generators in [16], but the convergence rate was not optimal. To prove the optimal rate $(0.3)$, we need hypotheses a bit stronger than those of [15], [16]. 
This paper, and several other related papers, grew out of a portion of the author's Ph.D thesis, which was written at the University of Chicago under the supervision of Jerry Bona. The author is grateful to Professors Jerry Bona, Jim Douglas, Ridgway Scott, and others for their suggestions and encouragement.

1. Nested Banach Spaces. A differential operator is usually viewed as a discontinuous operator from some Banach space $W^{m, p}(\Omega)$ into itself. But a differential operator of order $d$ may be continuous, or even Lipschitz or Fréchet differentiable, when viewed as a map from $W^{(k+1) d, p}(\Omega)$ into $W^{k d, p}(\Omega)$. Using several nested Banach spaces, $E_{3} \subseteq E_{2} \subseteq E_{1} \subseteq E_{0}$, we are able to treat some differential operators as if they were continuous or even differentiable, when they act on the solution $u(t)$ of our differential equation. Naturally, this requires us to make estimates of $|u(t)|_{3}$, where $\|_{3}$ is the norm of $E_{3}$; some mechanisms for keeping track of these estimates will be described in 2.4 and $2.8(\mathrm{c})$.

Differentiability may seem like a very strong condition; it is nearly the same as linearity. But it is not the same as linearity, and this will be reflected in our results. Proposition 5.9 is only proved for linear operators; that result does not generalize readily to a nonlinear setting. The theory of convergence of product formulas is much more complete for linear operators; see for instance [9].

1.1. Notations and Hypotheses. Let $\mathbf{R}_{+}=[0,+\infty)$. The maximum of two numbers $a, b \in \mathbf{R}_{+}$will be denoted by $a \vee b$.

A bounding function is a mapping $\gamma: \mathbf{R}_{+}^{2} \rightarrow[0,+\infty]$, nondecreasing in each argument, such that for each $r \in \mathbf{R}_{+}$there exists $t>0$ with $\gamma(t, r)<+\infty$.

Throughout this paper, we shall assume that $\left(E_{0}, \|_{0}\right),\left(E_{1}, \|_{1}\right),\left(E_{2}, \|_{2}\right),\left(E_{3}, \|_{3}\right)$ are Banach spaces. We assume $E_{3} \subseteq E_{2} \subseteq E_{1} \subseteq E_{0}$ with continuous inclusions, with $|f|_{j-1} \leqslant|f|_{j}$ for all $f \in E_{j}$. Also we assume that $\left(E_{3},||_{3}\right)$ is reflexive.

Let $\mathscr{L}$ be the Banach space of all continuous linear maps $\Lambda$ from $E_{1}$ into $E_{0}$, with norm $\|\Lambda\|_{\mathscr{L}} \equiv \sup \left\{|\Lambda f|_{0}:|f|_{1} \leqslant 1\right\}$.

1.2. Remarks. Bounding functions are so named because they will be used to specify bounds on various quantities. They play a role analogous to that of the generic constant $c$ of applied mathematics, or the notations $O(\cdot)$ and $o(\cdot)$ of asymptotic analysis.

If we use additional spaces $E_{4}, E_{5}$, etc., and more complicated approximation schemes, we can obtain still faster convergence rates by methods analogous to those of this paper; but the analysis is then much more complicated. We omit the details.

Most of the arguments in this paper are quantitative. However, our requirement that $\left(E_{3}, \|_{3}\right)$ be reflexive is a qualitative, or topological, condition, and so it seems less essential to the arguments of the paper. We shall use it in 1.3, below, which is used in turn to prove $2.7(\mathrm{~d}), 2.12,3.9,5.1$. It is not clear whether this qualitative condition can be replaced with a quantitative one. Property 1.3(a) is discussed further in [15].

1.3. Observations. Since $\left(E_{3},||_{3}\right)$ is reflexive, the sets $\left\{f \in E_{3}:|f|_{3} \leqslant r\right\}$ are weakly compact in $E_{3}$. From this we easily obtain the following two results:

(a) Extend ||$_{3}$ to a map defined on all of $E_{0}$, by taking $|f|_{3}=+\infty$ when $f \in$ $E_{0} \backslash E_{3}$. Then the mapping $\|_{3}: E_{0} \rightarrow[0,+\infty]$ is lower semicontinuous; i.e., the set $\left\{f \in E_{0} ;|f|_{3} \leqslant r\right\}$ is closed in $E_{0}$ for each $r \in \mathbf{R}_{+}$. 
(b) Suppose $u:[a, b] \rightarrow E_{3}$ is bounded, and $u$ is continuous when viewed as a map from $[a, b]$ into $E_{0}$. Then $|u(\cdot)|_{3}:[a, b] \rightarrow \mathbf{R}_{+}$is lower semicontinuous and bounded, and $u$ is continuous from $[a, b]$ to the weak topology on $E_{3}$. Hence $u$ is strongly measurable in $E_{3}$.

1.4. Definition. A mapping $W: E_{2} \rightarrow E_{0}$ is $\beta$-weakly regular if $\beta: \mathbf{R}_{+} \rightarrow \mathbf{R}_{+}$is a nondecreasing function, and for all $f, g \in E_{2}$ we have

$$
|W f|_{0} \leqslant \beta\left(|f|_{2}\right), \quad|W f-W g|_{0} \leqslant|f-g|_{2} \beta\left(|f|_{2} \vee|g|_{2}\right) .
$$

1.5. Definition. A mapping $A: E_{1} \rightarrow E_{0}$ is $\alpha$-regular if $\alpha: \mathbf{R}_{+} \rightarrow \mathbf{R}_{+}$is a nondecreasing function, and the following conditions both hold:

(a) $A\left(E_{j}\right) \subseteq E_{j-1}$ for $j=1,2,3$, and for all $f, g \in E_{j}$, we have

$$
|A f|_{j-1} \leqslant \alpha\left(|f|_{j}\right), \quad|A f-A g|_{j-1} \leqslant|f-g|_{j} \alpha\left(|f|_{j} \vee|g|_{j}\right) .
$$

(b) The mapping $A: E_{1} \rightarrow E_{0}$ is Fréchet differentiable; and for all $f, g \in E_{2} \subseteq E_{1}$, we have

$$
\left\|A^{\prime}(f)\right\|_{\mathscr{L}} \leqslant \alpha\left(|f|_{2}\right), \quad\left\|A^{\prime}(f)-A^{\prime}(g)\right\|_{\mathscr{L}} \leqslant|f-g|_{2} \alpha\left(|f|_{2} \vee|g|_{2}\right) .
$$

1.6. Remark. Some of the conditions in Definitions 1.4, 1.5 are redundant, in the sense that they could be omitted if we made a different choice of $\alpha, \beta$. For instance, if $W: E_{2} \rightarrow E_{0}$ satisfies

$$
|W f-W g|_{0} \leqslant|f-g|_{2} \beta\left(|f|_{2} \vee|g|_{2}\right) \quad \text { for all } f, g \in E_{2},
$$

where $\beta: \mathbf{R}_{+} \rightarrow \mathbf{R}_{+}$is some nondecreasing function, then it is easy to see that $W$ is $\tilde{\beta}$-weakly regular, where $\tilde{\beta}(r) \equiv \beta(r) \vee\left(|W(0)|_{0}+r \beta(r)\right)$. The redundant conditions are included as a convenience of notation; they will simplify computations later in this paper.

1.7. Lemma. Suppose $A$ is $\alpha$-regular. Then

$$
\left|A(f+t g)-A(f)-t A^{\prime}(f) g\right|_{0} \leqslant \frac{1}{2} t^{2}|g|_{1}|g|_{2} \alpha\left(|f|_{2}+t|g|_{2}\right)
$$

for all $f, g \in E_{2}$ and $t \geqslant 0$; and

$$
B(f) \equiv A^{\prime}(f) A(f) \quad \text { is }\left(2 \alpha^{2}\right) \text {-weakly regular } .
$$

Proof. To prove (1.8), first note that for $0 \leqslant s \leqslant t$ we have

$$
\begin{aligned}
\left\|A^{\prime}(f+s g)-A^{\prime}(f)\right\|_{\mathscr{L}} & \leqslant|(f+s g)-f|_{2} \alpha\left(|f+s g|_{2} \vee|f|_{2}\right) \\
& \leqslant s|g|_{2} \alpha\left(|f|_{2}+t|g|_{2}\right) .
\end{aligned}
$$

Now compute

$$
\begin{aligned}
\left|A(f+t g)-A(f)-t A^{\prime}(f) g\right|_{0} & =\left|\int_{0}^{t}\left\{\frac{d}{d s}[A(f+s g)]-A^{\prime}(f) g\right\} d s\right|_{0} \\
& =\left|\int_{0}^{t}\left\{A^{\prime}(f+s g) g-A^{\prime}(f) g\right\} d s\right|_{0} \\
& \leqslant \int_{0}^{t}\left\|A^{\prime}(f+s g)-A^{\prime}(f)\right\|_{\mathscr{L}}|g|_{1} d s,
\end{aligned}
$$


and now (1.8) follows immediately. To prove (1.9), compute

$$
|B f|_{0}=\left|A^{\prime}(f) A(f)\right|_{0} \leqslant\left\|A^{\prime}(f)\right\|_{\mathscr{L}}|A(f)|_{1} \leqslant \alpha\left(|f|_{2}\right)^{2}
$$

and

$$
\begin{aligned}
|B f-B g|_{0} & =\left|A^{\prime}(f) A(f)-A^{\prime}(g) A(g)\right|_{0} \\
& \leqslant\left.\left\|A^{\prime}(f)\right\|\right|_{\mathscr{L}}|A(f)-A(g)|_{1}+\left\|A^{\prime}(f)-A^{\prime}(g)\right\| \|_{\mathscr{L}}|A(g)|_{1} \\
& \leqslant \alpha\left(|f|_{2}\right)|f-g|_{2} \alpha\left(|f|_{2} \vee|g|_{2}\right)+|f-g|_{2} \alpha\left(|f|_{2} \vee|g|_{2}\right) \alpha\left(|g|_{2}\right) \\
& \leqslant 2|f-g|_{2} \alpha\left(|f|_{2} \vee|g|_{2}\right)^{2} .
\end{aligned}
$$

2. Semigroups and Semiflows. Let $\mathbf{R}_{+}=[0,+\infty)$. By a semigroup on a set $C$, we shall mean a family of mappings $S(t): C \rightarrow C\left(t \in \mathbf{R}_{+}\right)$, such that $S(0)=I$ and $S(t+s)=S(t) S(s)$. Semigroups arise naturally in the study of autonomous initial value problems. In general, if the problem

$$
u^{\prime}(t)=A u(t) \quad(t \geqslant 0), \quad u(0)=f,
$$

has a unique solution $u$ : $\mathbf{R}_{+} \rightarrow C$ for each $f \in C$, then the solution is given by $u(t)=S(t) f$ for some semigroup $S$ determined, or generated, by $A$. We say "in general" because we have glossed over some technicalities: the definition of "solution" varies from one paper to another according to the hypotheses on $A$. Some particular interpretations of "solution" will be given later in this section.

We shall usually denote by $e^{t A}$ the semigroup generated by $A$. This notation is natural: $e^{t A}$ is just the familiar exponential function $\sum_{0}^{\infty}(t A)^{j} / j !$ if $A$ is a real number, or more generally if $A$ is a continuous linear operator in a Banach space. That infinite sum may be meaningless for discontinuous, nonlinear operators $A$. But for many $A$ 's, continuous or not, linear or not, $\lim _{n \rightarrow \infty}(I-(t / n) A)^{-n}$ still exists and defines a semigroup $e^{t A}$; see [2], [5], [6]. We shall discuss that formula further in 6.6. For motivation, keep in mind that if $A$ is a partial differential operator, then $(I-t A)^{-1}$ may be an integral operator, and thus it may behave much better than $A$.

We shall use all three notations $S(t) f, u(t), e^{t A} f$. Each has its advantages, depending on what ideas we wish to emphasize. For instance, a Lie-Trotter product formula like (0.1) can be expressed in terms of initial value problems like (2.1): the solution of

$$
u^{\prime}(t)= \begin{cases}2 A u(t) & \text { when } 0<t<1 / n, \\ 2 B u(t) & \text { when } 1 / n<t<2 / n, \\ 2 A u(t) & \text { when } 2 / n<t<3 / n, \\ 2 B u(t) & \text { when } 3 / n<t<4 / n, \\ 2 A u(t) & \text { when } 4 / n<t<5 / n, \\ & \cdots\end{cases}
$$

converges to the solution of $u^{\prime}(t)=(A+B) u(t)$ when $n \rightarrow \infty$. This reformulation is less concise than (0.1), but it may help to explain our interest in (0.1): In some cases (2.2) may be easier to solve, or to compute numerically, or to analyze, than the equation $u^{\prime}(t)=(A+B) u(t)$. This may be the case if $A$ and $B$ are simple in 
different ways-e.g., if one is linear and the other is continuous, or if they operate on different spatial variables.

For some applications [1], a solution of (2.1) exists not on all of $\mathbf{R}_{+}$, but merely on some proper subinterval $[0, \tau)$. Hence we shall consider semiflows (i.e., local semigroups).

2.3. Definition. We shall say that $T$ is a semiflow on a set $C$ if the following four conditions hold:

(a) $(t, f) \mapsto T(t) f$ is a mapping from some domain $D(T) \subseteq \mathbf{R}_{+} \times C$, into $C$.

(b) For each $f \in C$, the set $D(T(\cdot) f) \equiv\left\{t \in \mathbf{R}_{+}:(t, f) \in D(T)\right\}$ is an interval containing 0 .

(c) $T(0) f=f$ for all $f \in C$.

(d) Let $t, s \geqslant 0$ and $f \in C$. Then $(t+s, f) \in D(T)$ if and only if both $(s, f)$ and $(t, T(s) f)$ belong to $D(T)$, in which case $T(t+s) f=T(t) T(s) f$.

As with semigroups, the semiflow generated by an operator $A$ will be denoted by $e^{t A}$. This will be made precise in the next few paragraphs.

2.4. Notation. We now introduce a particular type of semiflow on $\mathbf{R}_{+}$which will be needed for estimates later. Suppose that $\psi: \mathbf{R}_{+} \rightarrow \mathbf{R}_{+}$is locally Lipschitz and nondecreasing. Let $p_{0} \in \mathbf{R}_{+}$. Then the initial value problem

$$
p^{\prime}(t)=\psi(p(t)) \quad(0 \leqslant t<\tau), \quad p(0)=p_{0},
$$

has a unique solution, for some $\tau>0$. We shall denote that solution by $p(t)=$ $e^{t \psi(\cdot)} p_{0}=\exp [t \psi(\cdot)] p_{0}$. Note that $p(t)$ is a nondecreasing function of $t$. Choose $\tau$ as

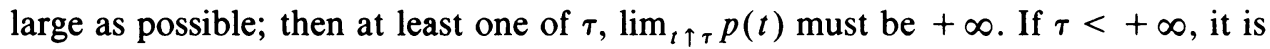
natural to define $p(t)=+\infty$ for all $t \geqslant \tau$. This extends the semiflow $e^{t \psi(\cdot)}$ on $\mathbf{R}_{+}$to a semigroup on $[0,+\infty]$. The inequality $\mathrm{e}^{r \psi(\cdot)} p_{0}<+\infty$ will be an abbreviation for the statement that $p_{0}<+\infty$ and $(2.5)$ has a finite solution on $[0, \tau)$ for some $\tau>r$.

Note that $\gamma(t, r) \equiv e^{t \psi(\cdot)} r$ is a bounding function (defined in 1.1).

2.6. Definition. Let $J \subseteq \mathbf{R}$ be an interval, and let $A: E_{1} \rightarrow E_{0}$ be some mapping such that $A\left(E_{3}\right) \subseteq E_{2}$. For the purposes of this paper, a solution of $u^{\prime}(t)=A u(t)$ $\left(t \in J\right.$ ) will mean a function $u: J \rightarrow E_{3}$ with these two properties:

(a) $|u(\cdot)|_{3}$ is bounded on compact subintervals of $J$.

(b) For all $a, b \in J$, the integral $\int_{a}^{b} A u(t) d t$ exists as a Bochner integral in $E_{2}$ and equals $u(b)-u(a)$.

2.7. Remarks. The Bochner integral is introduced in [10], [19]; in [8] it is simply called the integral. It follows from condition (b) above that

(c) considered as a mapping from $J$ into $E_{2}, u$ is absolutely continuous on compact subsets of $J$, and $u^{\prime}(t)$ exists and equals $A u(t)$ for almost all $t$ in $J$.

Hence, by Observation 1.3,

(d) $u: J \rightarrow E_{3}$ is weakly continuous, hence strongly measurable; and $|u(\cdot)|_{3}$ : $J \rightarrow \mathbf{R}_{+}$is lower semicontinuous and locally bounded.

2.8. Definition. For the purposes of this paper, we shall say that a mapping $A$ : $E_{1} \rightarrow E_{0}$ is a generator of type $(\alpha, \psi, \omega)$ if:

(a) $A$ is an $\alpha$-regular mapping from $E_{1}$ into $E_{0}$.

(b) $\psi: \mathbf{R}_{+} \rightarrow \mathbf{R}_{+}$and $\omega: \mathbf{R}_{+} \rightarrow \mathbf{R}$ are nondecreasing functions; $\psi$ is locally Lipschitz, and $\omega$ is continuous. 
(c) If $e^{\tau \psi(\cdot)}|f|_{3}<+\infty$, then there exists a solution of $u^{\prime}(t)=A u(t)$ on the interval $[0, \tau]$, satisfying $u(0)=f$ and

$$
|u(t)|_{3} \leqslant e^{t \psi(\cdot)}|f|_{3} \quad \text { for } t \in[0, \tau] .
$$

(d) If $u_{1}, u_{2}$ are solutions of $u^{\prime}(t)=A u(t)$ on some interval [0, $\tau$, then

$$
\left|u_{1}(\tau)-u_{2}(\tau)\right|_{0} \leqslant\left|u_{1}(0)-u_{2}(0)\right|_{0} \exp \left[\int_{0}^{\tau} \omega\left(\left|u_{1}(t)\right|_{3} \vee\left|u_{2}(t)\right|_{3}\right) d t\right] .
$$

2.9. Remarks. The solution $u(t)$ in 2.8 (c) will be denoted by $e^{t A} f$.

Condition $2.8(\mathrm{~d})$ guarantees that the solution $e^{t A} f$ is uniquely determined by $f$, and depends continuously on $f$ when $f$ is restricted to a ||$_{3}$-bounded set and $t$ is kept sufficiently small. The function $\omega$ plays a role analogous to the constant $\omega$ which appears in much of the literature on nonlinear semigroup theory [2], [3], [5], [6]. But a constant will not suffice for some applications; see the examples in [14], [15].

Note that $\alpha, \psi, \omega$ are not uniquely determined by $A$; they can easily be replaced by larger functions.

The arguments of this paper can be made to work with slightly weaker hypotheses on $\psi$ and $\omega$; see [15], [16] for similar arguments. But that would require longer definitions and proofs, so for brevity we shall use the conditions on $\psi$ and $\omega$ indicated above.

Some elementary examples of generators are given below. Additional characterizations of generators are given in 3.8, 3.9, 5.1. Deeper and more complicated examples, involving nonlinear partial differential equations with smooth coefficients, can be devised using estimates similar to those in [15]. Such long examples will be omitted from the present paper, however.

2.10. Example. Let $A$ be the generator of a strongly continuous, linear semigroup $e^{t A}$ on a Banach space $\left(E_{0}, \|_{0}\right)$, satisfying $\left\|e^{t A}\right\| \leqslant e^{t \omega}$ for some constant $\omega \in \mathbf{R}$. For $j=1,2,3$, let $E_{j}=D\left(A^{j}\right)$, with $|f|_{j}=|f|_{0}+|A f|_{0}+\cdots+\left|A^{j} f\right|_{0}$. Let $\psi(r) \equiv \omega r$, and let $\alpha=1$. Then $A$ is a generator of type $(\alpha, \psi, \omega)$. Note that $A^{\prime}(f) g=A g$ for all $f, g \in E_{1}$.

2.11. Another Example. Let $\left(E_{0},||_{0}\right)=\left(E_{1},\left.\right|_{1}\right)=\left(E_{2},\left.\right|_{2}\right)=\left(E_{3},||_{3}\right)$. Suppose $A: E_{0} \rightarrow E_{0}$ is continuously Fréchet differentiable, and $A^{\prime}: E_{0} \rightarrow \mathscr{L}$ is Lipschitz on bounded sets; i.e.,

$$
\left\|A^{\prime}(f)-A^{\prime}(g)\right\|_{\mathscr{L}} \leqslant|f-g|_{0} \varepsilon\left(|f|_{0} \vee|g|_{0}\right) \quad\left(f, g \in E_{0}\right)
$$

for some nondecreasing function $\varepsilon: \mathbf{R}_{+} \rightarrow \mathbf{R}_{+}$. Then $A$ is a generator of type $(\alpha, \psi, \omega)$, if those functions are selected as follows: First define

$$
\delta(r) \equiv \begin{cases}(1-r) \varepsilon(1)+(r-0) \varepsilon(2) & \text { when } 0 \leqslant r \leqslant 1 \\ (2-r) \varepsilon(2)+(r-1) \varepsilon(3) & \text { when } 1 \leqslant r \leqslant 2 \\ (3-r) \varepsilon(3)+(r-2) \varepsilon(4) & \text { when } 2 \leqslant r \leqslant 3 \\ \cdots & \end{cases}
$$

Then $\delta \geqslant \varepsilon$, and $\delta$ is nondecreasing and locally Lipschitz. Let $\omega(r)=\left\|A^{\prime}(0)\right\|_{\mathscr{L}^{+}}$ $r \delta(r)$ and $\psi(r)=|A(0)|_{0}+r \omega(r)$; then let $\alpha=\varepsilon \vee \psi \vee \omega$. We compute as follows:

$$
\left\|A^{\prime}(f)\right\|_{\mathscr{L}} \leqslant\left\|A^{\prime}(0)\right\|_{\mathscr{L}}+|f-0|_{0} \varepsilon\left(|f|_{0} \vee|0|_{0}\right) \leqslant \omega\left(|f|_{0}\right) ;
$$


hence

$$
\begin{aligned}
|A(f)-A(g)|_{0} & =\left|\int_{0}^{1}\left[\frac{d}{d t} A(t f+(1-t) g)\right] d t\right|_{0} \\
& =\left|\int_{0}^{1} A^{\prime}(t f+(1-t) g)(f-g) d t\right|_{0} \\
& \leqslant \int_{0}^{1}\left\|A^{\prime}(t f+(1-t) g)\right\|_{\mathscr{L}}|f-g|_{0} d t \leqslant \omega\left(|f|_{0} \vee|g|_{0}\right)|f-g|_{0} .
\end{aligned}
$$

This inequality yields condition $2.8(\mathrm{~d})$, by standard methods of ordinary differential equations. Next,

$$
|A(f)|_{0} \leqslant|A(0)|_{0}+|A(f)-A(0)|_{0} \leqslant|A(0)|_{0}+|f-0|_{0} \omega\left(|f|_{0} \vee|0|_{0}\right)=\psi\left(|f|_{0}\right) \text {. }
$$

This inequality yields condition 2.8 (c), again by standard methods. Since $\varepsilon, \psi, \omega \leqslant \alpha$, the above estimates also yield conditions 1.5. Thus $A$ is a generator of type $(\alpha, \psi, \omega)$.

2.12. LemMA. Suppose that $A, \alpha, \psi, \omega$ satisfy conditions $2.8(\mathrm{a}),(\mathrm{b})$. Also suppose there exists a bounding function $\delta$ with the following two properties:

(c') If $f \in E_{3}$ and $\tau \in \mathbf{R}_{+}$satisfy $\delta\left(\tau,|f|_{3}\right)<+\infty$, then there exists a solution of $u^{\prime}(t)=A u(t)$ with $u(0)=f$ and $|u(t)|_{3} \leqslant e^{t \psi(\cdot)}|f|_{3} \leqslant \delta\left(\tau,|f|_{3}\right)$, for all $t \in[0, \tau]$.

(d') If $\delta\left(\tau, \rho_{0}\right)<+\infty$ and if $u_{1}, u_{2}$ are solutions of $u^{\prime}(t)=A u(t)$ on $[0, \tau]$ with $\left|u_{1}(0)\right|_{3},\left|u_{2}(0)\right|_{3} \leqslant \rho_{0}$, then

$$
\left|u_{1}(\tau)-u_{2}(\tau)\right|_{0} \leqslant\left|u_{1}(0)-u_{2}(0)\right|_{0} \exp \left[\tau \omega\left(e^{\tau \psi(\cdot)} \rho_{0}\right)\right] .
$$

Then conditions 2.8(c), (d) also hold; that is, $A$ is a generator of type $(\alpha, \psi, \omega)$.

Proof. Let $u_{1}, u_{2}$ be any two solutions of $u^{\prime}(t)=A u(t)$ on an interval [0, $\left.\tau\right]$. Let $p(t) \equiv\left|u_{1}(t)\right|_{3} \vee\left|u_{2}(t)\right|_{3}$. Then $p:[0, \tau] \rightarrow \mathbf{R}_{+}$is bounded and lower semicontinuous, by $2.7(\mathrm{~d})$. Let $M=\sup \{p(t): 0 \leqslant t \leqslant \tau\}$. Since $\delta$ is a bounding function, there exists some $h>0$ such that $L \equiv \delta(h, M)$ is finite. Then $e^{h \psi(\cdot)} M \leqslant L$. By hypothesis $\left(\mathrm{d}^{\prime}\right)$, if $0 \leqslant a \leqslant b \leqslant \tau$ and $b-a \leqslant h$, then

$$
\left|u_{1}(b)-u_{2}(b)\right|_{0} \leqslant\left|u_{1}(a)-u_{2}(a)\right|_{0} \exp \left[(b-a) \omega\left(e^{(b-a) \psi(\cdot)} p(a)\right)\right] .
$$

The interval $[0, \tau]$ can be covered by finitely many such subintervals $[a, b]$. It follows that if $u_{1}(a)=u_{2}(a)$ for some $a \in[0, \tau]$, then $u_{1}=u_{2}$ on $[a, \tau]$. Thus, $u_{1}$ is uniquely determined on $[a, \tau]$ by its value at $a$.

By an application of hypothesis ( $\left.\mathbf{c}^{\prime}\right)$, there exists a solution of

$$
u^{\prime}(t)=A u(t) \quad(a \leqslant t \leqslant a+h), \quad u(a)=u_{1}(a),
$$

satisfying $|u(a+r)|_{3} \leqslant e^{r \psi(\cdot)}|u(a)|_{3}$ for all $r$ in $[0, h]$. But since $u_{1}$ is uniquely determined forward in time, we must have $u=u_{1}$. Again, the whole interval can be covered by finitely many subintervals of length less than or equal to $h$. Hence

$$
\left|u_{1}(t)\right|_{3} \leqslant e^{(t-s) \psi(\cdot)}\left|u_{1}(s)\right|_{3} \text {, whenever the right side is finite, }
$$

for $0 \leqslant s \leqslant t \leqslant \tau$. Similarly for $u_{2}$. Therefore $p(t) \leqslant e^{(t-s) \psi(\cdot)} p(s)$, whenever the right side is finite.

To prove 2.8(d), temporarily fix some integer $n$, large enough so that $\tau / n \leqslant h$. Let $a_{j}=j \tau / n$ for $j=0,1,2, \ldots, n$. Apply (2.13) to the interval $\left[a_{j-1}, a_{j}\right]$; we obtain

$$
\left|u_{1}\left(a_{j}\right)-u_{2}\left(a_{j}\right)\right|_{0} \leqslant\left|u_{1}\left(a_{j-1}\right)-u_{2}\left(a_{j-1}\right)\right|_{0} \exp \left[\frac{\tau}{n} \omega\left(e^{(\tau / n) \psi(\cdot)} p\left(a_{j-1}\right)\right)\right]
$$


for $j=1,2,3, \ldots, n$. Combining these $n$ inequalities, we obtain

$$
\begin{aligned}
\left|u_{1}(\tau)-u_{2}(\tau)\right|_{0} & \leqslant\left|u_{1}(0)-u_{2}(0)\right|_{0} \exp \left[\frac{\tau}{n} \sum_{j=1}^{n} \omega\left(e^{(\tau / n) \psi(\cdot)} p\left(a_{j-1}\right)\right)\right] \\
& \leqslant\left|u_{1}(0)-u_{2}(0)\right|_{0} \exp \left[\frac{\tau}{n} \sum_{j=0}^{n} \omega\left(e^{(\tau / n) \psi(\cdot)} p\left(a_{j}\right)\right)\right] \\
& \leqslant\left|u_{1}(0)-u_{2}(0)\right|_{0} \exp \left[\frac{\tau}{n} \omega\left(e^{(\tau / n) \psi(\cdot)} p\left(a_{0}\right)\right)+\int_{0}^{\tau} \omega\left(p_{n}(t)\right) d t\right],
\end{aligned}
$$

where $p_{n}$ is the step function which takes the value $e^{(\tau / n) \psi(\cdot)} p\left(a_{j}\right)$ on the interval $\left(a_{j-1}, a_{j}\right)$. Now, observe that $e^{(\tau / n) \psi(\cdot)} p\left(a_{0}\right) \rightarrow p\left(a_{0}\right)$ as $n \rightarrow \infty$, so

$$
(\tau / n) \omega\left(e^{(\tau / n) \psi(\cdot)} p\left(a_{0}\right)\right) \rightarrow 0 \text { when } n \rightarrow \infty .
$$

Also observe that if $t \in\left(a_{j-1}, a_{j}\right)$, then

$$
p_{n}(t)=e^{(\tau / n) \psi(\cdot)} p\left(a_{j}\right) \leqslant e^{(\tau / n) \psi(\cdot)} e^{\left(a_{j}-t\right) \psi(\cdot)} p(t) \leqslant e^{(2 \tau / n) \psi(\cdot)} p(t),
$$

and so $\lim \sup _{n \rightarrow \infty} p_{n}(t) \leqslant p(t)$. Since $\omega$ is continuous and nondecreasing, we have

$$
\limsup _{n \rightarrow \infty} \int_{0}^{\tau} \omega\left(p_{n}(t)\right) d t \leqslant \int_{0}^{\tau} \omega(p(t)) d t .
$$

This completes the proof of $2.8(\mathrm{~d})$.

To prove 2.8(c), let any $f \in E_{3}$ be given. Suppose that $\rho \equiv e^{\tau \psi(\cdot)}|f|_{3}$ is finite. Choose an integer $n$ large enough so that $\delta(\tau / n, \rho)<+\infty$. Using hypothesis $\left(\mathrm{c}^{\prime}\right)$, we show by induction on $j=0,1,2, \ldots, n$ that $u(t) \equiv e^{t A} f$ exists on $[0, j \tau / n]$ and satisfies $|u(t)|_{3} \leqslant e^{t \psi(\cdot)}|f|_{3}$ there. This completes the proof of the lemma.

3. Approximation Schemes. In this section we define a class of approximation schemes. In Propositions 3.8 and 3.9 and Theorem 5.1, we shall show that an operator $A$ has such a scheme $T$ if and only if $A$ is a generator, in which case $T(t / n)^{n} \rightarrow e^{t A}$ as $n \rightarrow \infty$.

3.1. Definition. We shall say that $T$ is an approximation scheme, restrained by rates $\psi, \omega$, for the evolution generated by $A$, correct of order 2 with remainder $W$ and bounding function $\gamma$-or more simply, we shall say that $(T, W, \psi, \omega, \gamma)$-if the following five conditions hold:

(a) $A: E_{1} \rightarrow E_{0}$ is an $\alpha$-regular mapping, for some $\alpha$; and $W: E_{3} \rightarrow E_{0}$ is a $\beta$-weakly regular mapping, for some $\beta$.

(b) $\psi: \mathbf{R}_{+} \rightarrow \mathbf{R}_{+}$and $\omega: \mathbf{R}_{+} \rightarrow \mathbf{R}$ are nondecreasing functions; $\psi$ is locally Lipschitz, and $\omega$ is continuous; $\gamma: \mathbf{R}_{+} \rightarrow \mathbf{R}_{+}$is a bounding function.

(c) $(t, f) \mapsto T(t) f$ is a mapping from some domain $D(T) \subseteq \mathbf{R}_{+} \times E_{3}$, into $E_{3}$.

(d) If $\gamma\left(t,|f|_{3}\right)<+\infty$, then $e^{t \psi(\cdot)}|f|_{3}<\gamma\left(t,|f|_{3}\right)$ and $(t, f) \in D(T)$, and

$$
\begin{gathered}
|T(t) f|_{3} \leqslant e^{t \psi(\cdot)}|f|_{3}, \\
|T(t) f-f|_{2} \leqslant t \gamma\left(t,|f|_{3}\right), \\
|T(t) f-f-t A f|_{1} \leqslant t^{2} \gamma\left(t,|f|_{3}\right), \\
\left|T(t) f-f-t A f-\frac{1}{2} t^{2} A^{\prime}(f) A(f)-\frac{1}{2} t^{2} W f\right|_{0} \leqslant t^{3} \gamma\left(t,|f|_{3}\right) .
\end{gathered}
$$

(e) Let $p_{0}=|f|_{3} \vee|g|_{3}$, and let $p_{1}=e^{t \psi(\cdot)} p_{0}$. If $\gamma\left(t, p_{0}\right)<+\infty$, then $p_{1}<+\infty$ and

$$
|T(t) f-T(t) g|_{0} \leqslant|f-g|_{0} \exp \left[t \omega\left(p_{1}\right)\right]
$$


We shall say that $T$ is a scheme for $A$ with remainder $W$ if there exist $\psi, \omega, \gamma$ such that $(T, W, \psi, \omega, \gamma)$ is a scheme for $A$.

3.7. Observations. (a) For each $T$, there is at most one $A$ satisfying (3.4).

(b) For each $T$ and $A$, there is at most one $W$ satisfying (3.5).

(c) Suppose $(T, W, \psi, \omega, \gamma)$ is a scheme for $A$, and $c \in \mathbf{R}_{+}$. Let $\tilde{c}=c \vee c^{3}$, let $\tilde{\gamma}(t, r) \equiv \tilde{c} \gamma(c t, r)$, and let $\tilde{T}(t) \equiv T(c t)$. Then $\left(\tilde{T}, c^{2} W, c \psi, c \omega, \tilde{\gamma}\right)$ is a scheme for $c A$.

(d) Suppose $T_{1}, T_{2}$ are schemes for the same operator $A$, with remainders $W_{1}, W_{2}$, respectively. Then $T(t) f \equiv \frac{1}{2}\left[T_{1}(t) f+T_{2}(t) f\right]$ defines a scheme for $A$ with remainder $W_{1}+W_{2}$.

3.8. Proposition. Let $A$ be a generator of type $(\alpha, \psi, \omega)$. Let $T(t) \equiv e^{t A}$. Then $(T, 0, \psi, \omega, \gamma)$ is a scheme for $A$, for some bounding function $\gamma$.

Proof. Let $\gamma_{1}(t, r) \equiv e^{t \psi(\cdot)} r$. Then whenever $\gamma_{1}\left(t,|f|_{3}\right)<+\infty$ we have $(t, f) \in$ $D(T)$ and $|T(t) f|_{3} \leqslant \gamma_{1}\left(t,|f|_{3}\right)$. Thus (3.2) is satisfied if $\gamma \geqslant \gamma_{1}$. Let $\gamma_{2}(t, r) \equiv$ $\alpha\left(\gamma_{1}(t, r)\right)$; then $\left|A\left(e^{t A} f\right)\right|_{2} \leqslant \gamma_{2}\left(t,|f|_{3}\right)$; hence

$$
|T(t) f-f|_{2}=\left|e^{t A} f-f\right|_{2}=\left|\int_{0}^{t} A\left(e^{s A} f\right) d s\right|_{2} \leqslant t \gamma_{2}\left(t,|f|_{3}\right) \text {. }
$$

Thus (3.3) is satisfied if $\gamma \geqslant \gamma_{2}$. Next, compute

$$
\begin{aligned}
|T(t) f-f-t A f|_{1} & =\left|\int_{0}^{t}\left[A\left(e^{s A} f\right)-A(f)\right] d s\right|_{1} \\
& \leqslant \int_{0}^{t}\left|A\left(e^{s A} f\right)-A(f)\right|_{1} d s \leqslant \int_{0}^{t}\left|e^{s A} f-f\right|_{2} \alpha\left(\left|e^{s A} f\right|_{2} \vee|f|_{2}\right) d s \\
& \leqslant \int_{0}^{t} s \gamma_{2}\left(s,|f|_{3}\right) \alpha\left(\gamma\left(s,|f|_{3}\right)\right) d s \leqslant \frac{1}{2} t^{2} \gamma_{2}\left(s,|f|_{3}\right)^{2}
\end{aligned}
$$

Thus (3.4) is satisfied if $\gamma \geqslant \frac{1}{2} \gamma_{2}^{2}$. Following (1.9), we have

$$
\begin{aligned}
\left|A^{\prime}\left(e^{t A} f\right) A\left(e^{t A} f\right)-A^{\prime}(f) A(f)\right|_{0} & \leqslant 2\left|e^{t A} f-f\right|_{2} \alpha\left(\left|e^{t A} f\right|_{2} \vee|f|_{2}\right)^{2} \\
& \leqslant 2 t \gamma_{2}\left(t,|f|_{3}\right)^{3}
\end{aligned}
$$

hence

$$
\begin{aligned}
\mid T(t) f & -f-t A f-\left.\frac{1}{2} t^{2} A^{\prime}(f) A(f)\right|_{0} \\
& =\left|\int_{0}^{t}\left[A\left(e^{s A} f\right)-A(f)\right] d s-\frac{1}{2} t^{2} A^{\prime}(f) A(f)\right|_{0} \\
& =\left|\int_{0}^{t} \int_{0}^{s}\left[\frac{d}{d r} A\left(e^{r A} f\right)\right] d r d s-\int_{0}^{t} \int_{0}^{s} A^{\prime}(f) A(f) d r d s\right|_{0} \\
& =\left|\int_{0}^{t} \int_{0}^{s}\left[A^{\prime}\left(e^{r A} f\right) A\left(e^{r A} f\right)-A^{\prime}(f) A(f)\right] d r d s\right|_{0} \\
& \leqslant \int_{0}^{t} \int_{0}^{s}\left|A^{\prime}\left(e^{r A} f\right) A\left(e^{r A} f\right)-A^{\prime}(f) A(f)\right|_{0} d r d s \\
& \leqslant \frac{1}{3} t^{3} \gamma_{2}\left(t,|f|_{3}\right)^{3} .
\end{aligned}
$$


Thus (3.5) is satisfied if $\gamma \geqslant \frac{1}{3} \gamma_{2}^{3}$. So $T$ is a scheme for $A$ with remainder 0 and bounding function $\gamma \equiv \max \left\{\gamma_{1}, \gamma_{2}, \frac{1}{2} \gamma_{2}^{2}, \frac{1}{3} \gamma_{2}^{3}\right\}$.

3.9. Proposition. Let $A$ be $\alpha$-regular. Let $(T, W, \psi, \omega, \gamma)$ be a scheme for $A$. Suppose that $T$ is a semiflow on $E_{3}$.

Then $A$ is a generator of type $(\alpha, \psi, \omega)$, and $T(t) \equiv e^{t A}$; hence $W=0$.

Proof. Let any $f \in E_{3}$ be given. Fix some $\tau>0$ small enough so that $\rho \equiv$ $\exp [\tau \psi(\cdot)]|f|_{3}$ is finite and $\gamma(\tau, \rho)$ is also finite. If $t, s \in[0, \tau]$, then

$$
\begin{aligned}
|T(t+s) f-T(s) f-t A T(s) f|_{1} & =|T(t) T(s) f-T(s) f-t A T(s) f|_{1} \\
& \leqslant t^{2} \gamma(\tau, \rho),
\end{aligned}
$$

hence $T(\cdot) f$ is differentiable in $E_{1}$ from the right at $s$, with derivative $A T(s) f$. Also,

$$
|T(t+s) f-T(s) f|_{2}=|T(t) T(s) f-T(s) f|_{2} \leqslant t \gamma(\tau, \rho),
$$

for $t, s \in[0, \tau]$. Hence

$$
\begin{aligned}
|A T(t+s) f-A T(s) f|_{1} & \leqslant|T(t+s) f-T(s) f|_{2} \alpha\left(|T(t+s) f|_{2} \vee|T(s) f|_{2}\right) \\
& \leqslant t \gamma(\tau, \rho) \alpha(\rho),
\end{aligned}
$$

so the map $s \rightarrow A T(s) f$ is continuous into $E_{1}$. Also, if $0 \leqslant h \leqslant s \leqslant \tau$,

$$
\begin{aligned}
\mid T(s- & h) f-T(s) f+\left.h A T(s) f\right|_{1} \\
= & |T(s-h) f-T(h) T(s-h) f+h A T(s) f|_{1} \\
\leqslant & |T(h) T(s-h) f-T(s-h) f-h A T(s-h) f|_{1} \\
& +h|A T(s-h) f-A T(s) f|_{1} \\
\leqslant & h^{2} \gamma(\tau, \rho)(1+\alpha(\rho)),
\end{aligned}
$$

so $T(\cdot) f$ is also differentiable in $E_{1}$ from the left at $s$, with derivative $A T(s) f$.

Thus the mapping $T(\cdot) f:[0, \tau] \rightarrow E_{1}$ is continuously differentiable, with derivative $(d / d s) T(s) f=A T(s) f$. Hence it satisfies this integral equation in $E_{1}$ :

$$
T(t) f=f+\int_{0}^{t} A T(s) f d s .
$$

Since $T(\cdot) f$ is bounded in $E_{3}$ and continuous in $E_{0}$, it is strongly measurable in $E_{3}$, by Observation 1.3(b). Therefore $A(T(\cdot) f):[0, \tau] \rightarrow E_{2}$ is bounded and strongly measurable; hence it is Bochner-integrable. Thus Eq. (3.10) holds in $E_{2}$.

By Lemma 2.12, it follows that $A$ is a generator of type $(\alpha, \psi, \omega)$, and $T(t) \equiv e^{t A}$. By Observation 3.7(b) and Proposition 3.8, we have $W=0$.

\section{Composition of Approximation Schemes.}

4.1. THEOREM. If $T_{1}, T_{2}$ are schemes for $A_{1}, A_{2}$, respectively, then $T(t) \equiv T_{1}(t) T_{2}(t)$ is a scheme for $A \equiv A_{1}+A_{2}$. More precisely: For $j=1$, 2, suppose that $A_{j}$ is $\alpha_{j}$-regular. $W_{j}$ is $\beta_{j}$-weakly regular, and $\left(T_{j}, W_{j}, \psi_{j}, \omega_{j}, \gamma_{j}\right)$ is a scheme for $A_{j}$. Let $\alpha=\alpha_{1}+\alpha_{2}$,

$$
W(f)=W_{1}(f)+W_{2}(f)+\left[A_{1}^{\prime}(f) A_{2}(f)-A_{2}^{\prime}(f) A_{1}(f)\right],
$$


$\beta(r)=\beta_{1}(r)+\beta_{2}(r)+4 \alpha_{1}(r) \alpha_{2}(r), \psi=2\left(\psi_{1} \vee \psi_{2}\right)$, and $\omega=\omega_{1}+\omega_{2}$. Then: $A$ is $\alpha$-regular, $W$ is $\beta$-regular, and $(T, W, \psi, \omega, \gamma)$ is a scheme for $A$, where the bounding function $\gamma$ can be chosen as follows: Let

$$
\nu \equiv \nu(t, r) \equiv t \alpha_{2}(r)+\exp \left[t \psi_{2}(\cdot)\right] r
$$

Then let

$$
\begin{aligned}
\gamma(t, r) \equiv & \gamma_{2}(t, r)\left[1+\alpha_{1}(\nu)+\alpha_{1}(\nu)^{2}+\frac{1}{2} \beta_{2}(\nu)\right] \\
& +\gamma_{1}(t, \nu)+\frac{1}{2} \alpha_{1}(\nu) \alpha_{2}(r)^{2} .
\end{aligned}
$$

Proof. Obviously $A$ is $\alpha$-regular; and $W$ is $\beta$-weakly regular by an argument similar to the proof of (1.9). The verifications of (3.2) and (3.6) are easy and are omitted. To verify that $T, A, \gamma$ satisfy (3.3), compute

$$
\begin{aligned}
|T(t) f-f|_{2} & =\left|T_{1}(t) T_{2}(t) f-f\right|_{2} \leqslant\left|T_{1}(t) T_{2}(t) f-T_{2}(t) f\right|_{2}+\left|T_{2}(t) f-f\right|_{2} \\
& \leqslant t \gamma_{1}\left(t,\left|T_{2}(t) f\right|_{3}\right)+t \gamma_{2}\left(t,|f|_{3}\right) \\
& \leqslant t \gamma_{1}\left(t, e^{t \psi_{2}(\cdot)}|f|_{3}\right)+t \gamma_{2}\left(t,|f|_{3}\right) \leqslant t \gamma\left(t,|f|_{3}\right) .
\end{aligned}
$$

To verify that $T, A, \gamma$ satisfy (3.4), we first compute

$$
\begin{aligned}
\left|A_{1} T_{2}(t) f-A_{1} f\right|_{1} & \leqslant\left|T_{2}(t) f-f\right|_{2} \alpha_{1}\left(\left|T_{2}(t) f\right|_{2} \vee|f|_{2}\right) \\
& \leqslant t \gamma_{2}\left(t,|f|_{3}\right) \alpha_{1}\left(\nu\left(t,|f|_{3}\right)\right) .
\end{aligned}
$$

Then we use that estimate, together with the fact that $T_{j}, A_{j}, \gamma_{j}$ satisfy (3.4) for $j=1,2$; we obtain

$$
\begin{aligned}
\mid T(t) f & -f-\left.t A f\right|_{1}=\left|T_{1}(t) T_{2}(t) f-f-t A_{1} f-t A_{2} f\right|_{1} \\
\leqslant & \left|T_{1}(t) T_{2}(t) f-T_{2}(t) f-t A_{1} T_{2}(t) f\right|_{1} \\
& \quad+\left|T_{2}(t) f-f-t A_{2} f\right|_{1}+t\left|A_{1} T_{2}(t) f-A_{1} f\right|_{1} \\
\leqslant & t^{2} \gamma_{1}\left(t,\left|T_{2}(t) f\right|_{3}\right)+t^{2} \gamma_{2}\left(t,|f|_{3}\right)+t^{2} \gamma_{2}\left(t,|f|_{3}\right) \alpha_{1}\left(\nu\left(t,|f|_{3}\right)\right) \\
\leqslant & t^{2} \gamma\left(t,|f|_{3}\right) .
\end{aligned}
$$

The verification of (3.5) will take longer. We perform several preliminary computations. First, following (1.9),

$$
\begin{aligned}
& \left|A_{1}^{\prime}\left(T_{2}(t) f\right) A_{1}\left(T_{2}(t) f\right)-A_{1}^{\prime}(f) A_{1}(f)\right|_{0} \\
& \quad \leqslant 2\left|T_{2}(t) f-f\right|_{2} \alpha_{1}\left(|f|_{2} \vee\left|T_{2}(t) f\right|_{2}\right)^{2} \leqslant 2 t \gamma_{2}\left(t,|f|_{3}\right) \alpha_{1}\left(\nu\left(t,|f|_{3}\right)\right)^{2} .
\end{aligned}
$$

Second, using 1.4,

$$
\begin{aligned}
\left|W_{2}\left(T_{2}(t) f\right)-W_{2}(f)\right|_{0} & \leqslant\left|T_{2}(t) f-f\right|_{2} \beta_{2}\left(\left|T_{2}(t) f\right|_{2} \vee|f|_{2}\right) \\
& \leqslant t \gamma_{2}\left(t,|f|_{3}\right) \beta_{2}\left(\nu\left(t,|f|_{3}\right)\right) .
\end{aligned}
$$

Third, using 1.5(a),

$$
\begin{aligned}
& \left|A_{1}\left(T_{2}(t) f\right)-A_{1}\left(f+t A_{2}(f)\right)\right|_{0} \\
& \quad \leqslant\left|T_{2}(t) f-\left(f+t A_{2} f\right)\right|_{1} \alpha_{1}\left(\left|T_{2}(t) f\right|_{1} \vee\left|f+t A_{2}(f)\right|_{1}\right) \\
& \quad \leqslant t^{2} \gamma_{2}\left(t,|f|_{3}\right) \alpha_{1}\left(\left[\left|T_{2}(t) f\right|_{1} \vee|f|_{1}\right]+t \alpha_{2}\left(|f|_{2}\right)\right) \\
& \quad \leqslant t^{2} \gamma_{2}\left(t,|f|_{3}\right) \alpha_{1}\left(\nu\left(t,|f|_{3}\right)\right)
\end{aligned}
$$


Fourth, following (1.8),

$$
\begin{aligned}
& \left|A_{1}\left(f+t A_{2}(f)\right)-A_{1}(f)-t A_{1}^{\prime}(f) A_{2}(f)\right|_{0} \\
& \quad \leqslant \frac{1}{2} t^{2}\left|A_{2}(f)\right|_{2}^{2} \alpha_{1}\left(|f|_{2}+t\left|A_{2}(f)\right|_{2}\right) \leqslant \frac{1}{2} t^{2} \alpha_{2}\left(|f|_{3}\right)^{2} \alpha_{1}\left(\nu\left(t,|f|_{3}\right)\right) .
\end{aligned}
$$

We now use the four estimates above, plus the fact that $T_{j}, A_{j}, W_{j}, \gamma_{j}$ satisfy (3.5) for $j=1,2$. We obtain

$$
\begin{aligned}
\mid T(t) f- & f-t A f-\frac{1}{2} t^{2} A^{\prime}(f) A(f)-\left.\frac{1}{2} t^{2} W(f)\right|_{0} \\
= & \mid T_{1}(t) T_{2}(t) f-f-t A_{1} f-t A_{2} f \\
& \quad-\frac{1}{2} t^{2}\left[A_{1}^{\prime}(f)+A_{2}^{\prime}(f)\right]\left[A_{1}(f)+A_{2}(f)\right] \\
& \quad-\left.\frac{1}{2} t^{2}\left[W_{1} f+W_{2} f+A_{1}^{\prime}(f) A_{2}(f)-A_{2}^{\prime}(f) A_{1}(f)\right]\right|_{0} \\
= & T_{1}(t) T_{2}(t) f-f-t A_{1} f-t A_{2} f \\
& \quad-\left.\frac{1}{2} t^{2}\left[W_{1} f+W_{2} f+2 A_{1}^{\prime}(f) A_{2}(f)+A_{1}^{\prime}(f) A_{1}(f)+A_{2}^{\prime}(f) A_{2}(f)\right]\right|_{0} \\
\leqslant & T_{1}(t) T_{2}(t) f-T_{2}(t) f-t A_{1}\left(T_{2}(t) f\right) \\
& \quad-\frac{1}{2} t^{2} A_{1}^{\prime}\left(T_{2}(t) f\right) A_{1}\left(T_{2}(t) f\right)-\left.\frac{1}{2} t^{2} W_{2}\left(T_{2}(t) f\right)\right|_{0} \\
& +\left.\frac{1}{2} t^{2}\left|W_{1}\left(T_{2}(t) f\right) A_{1}\left(T_{2}(t) f\right)-W_{2}(f)\right|_{0}^{\prime}(f) A_{1}(f)\right|_{0} \\
& +\left|T_{2}(t) f-f-t A_{2} f-\frac{1}{2} t^{2} A_{2}^{\prime}(f) A_{2}(f)-\frac{1}{2} t^{2} W_{1}(f)\right|_{0} \\
& +t\left|A_{1}\left(T_{2}(t) f\right)-A_{1}\left(f+t A_{2}(f)\right)\right|_{0} \\
& +t\left|A_{1}\left(f+t A_{2}(f)\right)-A_{1}(f)-t A_{1}^{\prime}(f) A_{2}(f)\right|_{0} \\
\leqslant & t^{3} \gamma_{1}\left(t, \nu\left(t,|f|_{3}\right)\right)+t^{3} \gamma_{2}\left(t,|f|_{3}\right) \alpha_{1}\left(\nu\left(t,|f|_{3}\right)\right)^{2} \\
& +\frac{1}{2} t^{3} \gamma_{2}\left(t,|f|_{3}\right) \beta_{2}\left(\nu\left(t,|f|_{3}\right)\right) \\
& +t^{3} \gamma_{2}\left(t,|f|_{3}\right)+t^{3} \gamma_{2}\left(t,|f|_{3}\right) \alpha_{1}\left(\nu\left(t,|f|_{3}\right)\right) \\
& +\frac{1}{2} t^{3} \alpha_{2}\left(|f|_{3}\right)^{2} \alpha_{1}\left(\nu\left(t,|f|_{3}\right)\right) \\
\leqslant & \gamma\left(t,|f|_{3}\right)
\end{aligned}
$$

This completes the proof of the theorem.

4.5. Corollary. For $j=1,2,3, \ldots$, suppose that $T_{j}$ is a scheme for $A_{j}$ with remainder $W_{j}$. Then $T(t)=T_{n}(t) T_{n-1}(t) \cdots T_{2}(t) T_{1}(t)$ is a scheme for $A_{1}+A_{2}+$ $\cdots+A_{n}$ with remainder

$$
W(f)=\sum_{1 \leqslant j \leqslant n} W_{j}(f)+\sum_{1 \leqslant j<k \leqslant n}\left\{A_{k}^{\prime}(f) A_{j}(f)-A_{j}^{\prime}(f) A_{k}(f)\right\} ;
$$

and

$$
\hat{T}(t) \equiv \frac{1}{2}\left\{T_{1}(t) T_{2}(t) \cdots T_{n-1}(t) T_{n}(t)+T_{n}(t) T_{n-1}(t) \cdots T_{2}(t) T_{1}(t)\right\}
$$

is a scheme for $A_{1}+A_{2}+\cdots+A_{n}$ with remainder $\sum_{j=1}^{n} W_{j}(f)$.

Proof. The first conclusion follows from Theorem 4.1 by induction on $n$. The second conclusion then follows from Observation 3.7(d). 
4.6. TheOREM. Suppose $A$ is $\alpha$-regular, $W$ is $\beta$-weakly regular, and $(T, W, \psi, \omega, \gamma)$ is a scheme for $A$. Let $n$ be a positive integer, and let $U(t) \equiv T(t / n)^{n}$. Then $\left(U, n^{-1} W, \psi, \omega, \delta\right)$ is a scheme for $A$, for some bounding function $\delta$; and $\delta$ can be chosen independent of $n$. In fact, we can choose $\delta$ as follows: Let

$$
\nu \equiv \nu(t, r) \equiv t \alpha(r)+e^{t \psi(\cdot)} r
$$

then let

$$
\delta(t, r) \equiv\left[\gamma(t, \nu)+\frac{1}{2} \alpha(\nu)^{3}\right] \exp \left[\alpha(\nu)+\alpha(\nu)^{2}+\frac{1}{2} \beta(\nu)\right]
$$

\section{Proof. Define}

$$
b \equiv b(t, r) \equiv \alpha(\nu)+\alpha(\nu)^{2}+\frac{1}{2} \beta(\nu), \quad c \equiv c(t, r) \equiv \gamma(t, \nu)+\frac{1}{2} \alpha(\nu)^{3} .
$$

Then $\delta(t, r)=e^{b} c$, and these quantities are independent of $n$.

Fix any $n$. Let $U_{j}(t) \equiv T(t / n)^{j}(j=0,1,2, \ldots, n)$, with $U_{0}(t) \equiv I$. We easily verify that $U_{j}, j n^{-1} \psi, j n^{-1} \omega$ satisfy (3.2) and (3.6). Also, by Observation 3.7(c), $\left(U_{1}, n^{-2} W, n^{-1} \psi, n^{-1} \omega, n^{-1} \gamma\right)$ is a scheme for $n^{-1} A$. We proceed by induction: for some $k \in\{0,1,2, \ldots, n-1\}$, assume that $\left(U_{k}, k n^{-2} W, k n^{-1} \psi, k n^{-1} \omega, \varepsilon_{k}\right)$ is a scheme for $k^{-1} n A$ with some bounding function $\varepsilon_{k}$. (This is trivial for $k=0$, with $\varepsilon_{0}=0$.) We shall apply Theorem 4.1, with

$$
\begin{array}{lll}
A_{1}=n^{-1} A, & W_{1}=n^{-2} W, & T_{1}=U_{1}, \\
A_{2}=k n^{-1} A, & W_{2}=k n^{-2} W, & T_{2}=U_{k}, \\
\alpha_{1}=n^{-1} \alpha, & \beta_{1}=n^{-2} \beta, & \gamma_{1}=n^{-1} \gamma, \\
\alpha_{2}=\alpha, & \beta_{2}=k n^{-2} \beta, & \gamma_{2}=\varepsilon_{k} .
\end{array}
$$

We could apply that theorem with $\alpha_{2}=k n^{-1} \alpha$ and $\psi_{2}=k n^{-1} \psi$. But we shall instead use the slightly larger functions $\alpha_{2} \equiv \alpha$ and $\psi_{2} \equiv \psi$; these have the advantage of being independent of $k$ and $n$. Note that our definition of $\nu$ is consistent with the definition used in Theorem 4.1.

Observe that $U_{k+1}(t)=U_{1}(t) U_{k}(t)$. By Theorem 4.1, $U_{k+1}$ is a scheme for $(k+1) n^{-1} A$, with remainder $(k+1) n^{-2} W$, and with bounding function $\varepsilon_{k+1}$ given by formulas (4.3)-(4.4). That is,

$$
\begin{array}{r}
\varepsilon_{k+1}(t, r) \equiv \gamma_{2}(t, r)\left[1+\alpha_{1}(\nu)+\alpha_{1}(\nu)^{2}+\frac{1}{2} \beta_{2}(\nu)\right]+\gamma_{1}(t, \nu)+\frac{1}{2} \alpha_{1}(\nu) \alpha_{2}(r)^{2} \\
=\varepsilon_{k}(t, r)\left[1+\frac{\alpha(\nu)}{n}+\left(\frac{\alpha(\nu)}{n}\right)^{2}+\frac{k \beta(\nu)}{2 n^{2}}\right]+\frac{\gamma(t, \nu)}{n}+\frac{1}{2}\left(\frac{\alpha(\nu)}{n}\right) \alpha(r)^{2} .
\end{array}
$$

In this fashion we recursively define bounding functions $\varepsilon_{1}, \varepsilon_{2}, \ldots, \varepsilon_{n}$. From the recursion formula above it follows that $\varepsilon_{k+1} \leqslant(1+(b / n)) \varepsilon_{k}+(c / n)$, with $b, c$ defined as in (4.7). Solve this inequality recursively, with initial value $\varepsilon_{0}=0$; we obtain

$$
\begin{aligned}
\varepsilon_{k} & \leqslant\left(1+\frac{b}{n}\right)^{k-1} \frac{c}{n}+\left(1+\frac{b}{n}\right)^{k-2} \frac{c}{n}+\cdots+\left(1+\frac{b}{n}\right) \frac{c}{n}+\frac{c}{n} \\
& =\left[\left(1+\frac{b}{n}\right)^{k}-1\right] \frac{c}{b} .
\end{aligned}
$$

Finally, for $k=n$ we obtain

$$
\varepsilon_{n} \leqslant\left[\left(1+\frac{b}{n}\right)^{n}-1\right] \frac{c}{b} \leqslant\left(e^{b}-1\right) \frac{c}{b} \leqslant e^{b} c=\delta(t, r) .
$$

This completes the proof of the theorem. 


\section{Convergence Rates.}

5.1. TheOREM. Suppose $A$ is $\alpha$-regular, $W$ is $\beta$-weakly regular, and $(T, W, \psi, \omega, \gamma)$ is a scheme for $A$.

Then $A$ is a generator of type $(\alpha, \psi, \omega)$, and $e^{t A}$ can be approximated as follows: Define $\delta$ as in Theorem 4.6, and let $\rho=e^{t \psi(\cdot)}|f|_{3}$. Then

$$
\left|T\left(\frac{t}{n}\right)^{n} f-e^{t A} f\right|_{0} \leqslant \frac{2 t^{2}}{n} \delta(t, \rho) e^{t \omega(\rho)},
$$

whenever $\rho<+\infty$ and $\delta(t, \rho)<+\infty$. Moreover, if $W=0$, then

$$
\left|T\left(\frac{t}{n}\right)^{n} f-e^{t A} f\right|_{0} \leqslant \frac{2 t^{3}}{n^{2}} \delta(t, \rho) e^{t \omega(\rho)} .
$$

Proof. For positive integers $n$, let $U_{n}(t) \equiv T(t / n)^{n}$. By Theorem 4.6, whenever $\delta\left(t,|f|_{3} \vee|g|_{3}\right)<+\infty$, we have

$$
\begin{gathered}
\left|U_{n}(t) f\right|_{3} \leqslant e^{t \psi(\cdot)}|f|_{3}, \\
\left|U_{n}(t) f-f\right|_{2} \leqslant t \delta\left(t,|f|_{3}\right), \\
\left|U_{n}(t) f-f-t A f\right|_{1} \leqslant t^{2} \delta\left(t,|f|_{3}\right), \\
\left|U_{n}(t) f-f-t A f-\frac{1}{2} t^{2} A^{\prime}(f) A(f)-\frac{1}{2} t^{2} n^{-1} W f\right|_{0} \leqslant t^{3} \delta\left(t,|f|_{3}\right), \\
\left|U_{n}(t) f-U_{n}(t) g\right|_{0} \leqslant|f-g|_{0} \exp \left[t \omega\left(e^{t \psi(\cdot)}\left(|f|_{3} \vee|g|_{3}\right)\right)\right] .
\end{gathered}
$$

Now let

$$
\lambda= \begin{cases}1 & \text { if } W \neq 0 \\ 2 & \text { if } W=0\end{cases}
$$

Since $|g|_{0} \leqslant|g|_{1}$ for $g \in E_{1}$, from (5.6)-(5.7) we obtain.

$$
\left|U_{n}(t) f-U_{m}(t) f\right|_{0} \leqslant 2 t^{1+\lambda} \delta\left(t,|f|_{3}\right)
$$

for all positive integers $m, n$. Let $\rho=\rho\left(t,|f|_{3}\right)=e^{t \psi(\cdot)}|f|_{3}$. Then

$$
\begin{aligned}
\left|U_{n k}(t) f-U_{k}(t) f\right|_{0}=\left|U_{n}\left(\frac{t}{k}\right)^{k} f-U_{1}\left(\frac{t}{k}\right)^{k} f\right|_{0} \\
=\left|\sum_{j=1}^{k}\left[U_{n}\left(\frac{t}{k}\right)^{k-j} U_{n}\left(\frac{t}{k}\right) U_{1}\left(\frac{t}{k}\right)^{j-1} f-U_{n}\left(\frac{t}{k}\right)^{k-j} U_{1}\left(\frac{t}{k}\right) U_{1}\left(\frac{t}{k}\right)^{j-1} f\right]\right|_{0} \\
\leqslant \sum_{j=1}^{k}\left|U_{n}\left(\frac{t}{k}\right) U_{1}\left(\frac{t}{k}\right)^{j-1} f-U_{1}\left(\frac{t}{k}\right) U_{1}\left(\frac{t}{k}\right)^{j-1} f\right|_{0} \\
\quad \cdot \exp \left\{\frac { k - j } { k } t \omega \left(e^{\left.\left.(j / k) t \psi(\cdot)|f|_{3}\right)\right\}}\right.\right. \\
\leqslant \sum_{j=1}^{k} 2\left(\frac{t}{k}\right)^{1+\lambda} \delta\left(\frac{t}{k},\left|U_{1}\left(\frac{t}{k}\right)^{j-1} f\right|_{3}\right) e^{t \omega(\rho)} \leqslant \frac{2 t^{1+\lambda}}{k^{\lambda}} \delta(t, \rho) e^{t \omega(\rho)} .
\end{aligned}
$$

Then, for any positive integers $m, n$,

$$
\begin{aligned}
\left|U_{n}(t) f-U_{m}(t) f\right|_{0} & \leqslant\left|U_{n}(t) f-U_{n m}(t) f\right|_{0}+\left|U_{n m}(t) f-U_{m}(t) f\right|_{0} \\
& \leqslant 2 t^{1+\lambda}\left(1 / n^{\lambda}+1 / m^{\lambda}\right) \delta(t, \rho) e^{t \omega(\rho)}
\end{aligned}
$$


It follows that $\left\{U_{n}(t) f\right\}_{n=1}^{\infty}$ converges in $E_{0}$ to some limit $S(t) f$, with convergence rate

$$
\left|S(t) f-U_{n}(t) f\right|_{0} \leqslant \frac{2 t^{1+\lambda}}{n^{\lambda}} \delta(t, \rho) e^{t \omega(\rho)},
$$

whenever $\rho<+\infty$ and $\delta<+\infty$.

Since $\left|U_{n}(t) f\right|_{3} \leqslant \rho$ for all $n$, the sequence $\left\{U_{n}(t) f\right\}_{n=1}^{\infty}$ is relatively weakly compact in the reflexive space $E_{3}$. Therefore $S(t) f \in E_{3}$, and $U_{n}(t) f \rightarrow S(t) f$ weakly in $E_{3}$. Hence $U_{n}(t) f \rightarrow S(t) f$ weakly also in $E_{2}$ and $E_{1}$. Taking limits, we find that all of inequalities (5.4)-(5.6) and (5.8) remain valid when $U_{n}$ is replaced by $S$; and in the limit, (5.7) yields

$$
\left|S(t) f-f-t A f-\frac{1}{2} t^{2} A^{\prime}(f) A(f)\right|_{0} \leqslant t^{3} \delta\left(t,|f|_{3}\right) .
$$

Thus $(S, 0, \psi, \omega, \delta)$ is a scheme for $A$.

When $0 \leqslant \sigma \leqslant \tau$, then

$$
\begin{aligned}
|T(\tau) f-T(\sigma) f|_{0} & \leqslant|T(\tau) f-f-\tau A f|_{1}+|T(\sigma) f-f-\sigma A f|_{1}+(\tau-\sigma)|A f|_{1} \\
& \leqslant 2 \tau^{2} \gamma\left(\tau,|f|_{3}\right)+(\tau-\sigma) \alpha\left(|f|_{3}\right) .
\end{aligned}
$$

Hence, if $0 \leqslant s \leqslant t$, we have

$$
\begin{aligned}
& \left|U_{n}(t) f-U_{n}(s) f\right|_{0}=\left|T\left(\frac{t}{n}\right)^{n} f-T\left(\frac{s}{n}\right)^{n} f\right|_{0} \\
& =\left|\sum_{j=1}^{n}\left(T\left(\frac{t}{n}\right)^{n-j} T\left(\frac{t}{n}\right) T\left(\frac{s}{n}\right)^{j-1} f-T\left(\frac{t}{n}\right)^{n-j} T\left(\frac{s}{n}\right) T\left(\frac{s}{n}\right)^{j-1} f\right)\right|_{0} \\
& \quad \leqslant e^{t \omega(\rho)} \sum_{j=1}^{n}\left|T\left(\frac{t}{n}\right) T\left(\frac{s}{n}\right)^{j-1} f-T\left(\frac{s}{n}\right) T\left(\frac{s}{n}\right)^{j-1} f\right|_{0} \\
& \leqslant e^{t \omega(\rho)} \sum_{j=1}^{n}\left\{2\left(\frac{t}{n}\right)^{2} \gamma(t, \rho)+\frac{t-s}{n} \alpha(\rho)\right\} \\
& =e^{t \omega(\rho)}\left[2 \frac{t^{2}}{n} \gamma(t, \rho)+(t-s) \alpha(\rho)\right],
\end{aligned}
$$

whenever $\gamma(t, \rho)<+\infty$. Taking limits,

$$
|S(t) f-S(s) f|_{0} \leqslant(t-s) e^{t \omega(\rho)} \alpha(\rho),
$$

and so the mapping $t \mapsto S(t) f$ is continuous.

Let any $f \in E_{3}$ and any positive integers $m, n$ be given. Choose $t>0$ small enough so that $\mu \equiv e^{(m+n) t \psi(\cdot)}|f|_{3}$ is finite and also $\delta((m+n) t, \mu)$ is finite. As $k \rightarrow \infty$, we have the following convergences in $E_{0}$ :

$$
\begin{aligned}
T\left(\frac{t}{k}\right)^{(m+n) k} f & =T\left(\frac{(m+n) t}{(m+n) k}\right)^{(m+n) k} f \rightarrow S((m+n) t) f, \\
T\left(\frac{t}{k}\right)^{m k} S(n t) f & =T\left(\frac{m t}{m k}\right)^{m k} S(n t) f \rightarrow S(m t) S(n t) f, \\
T\left(\frac{t}{k}\right)^{n k} f & =T\left(\frac{n t}{n k}\right)^{n k} f \rightarrow S(n t) f .
\end{aligned}
$$


Also,

$$
\begin{aligned}
\left|T\left(\frac{t}{k}\right)^{(m+n) k} f-T\left(\frac{t}{k}\right)^{m k} S(n t) f\right|_{0} \\
\quad=\left|T\left(\frac{t}{k}\right)^{m k} T\left(\frac{t}{k}\right)^{n k} f-T\left(\frac{t}{k}\right)^{m k} S(n t) f\right|_{0} \\
\leqslant e^{m t \omega(\mu)}\left|T\left(\frac{t}{k}\right)^{n k} f-S(n t) f\right|_{0} \rightarrow 0 .
\end{aligned}
$$

Hence $S((m+n) t) f=S(m t) S(n t) f$ for integers $m$ and $n$. Therefore $S(\tau+\sigma)=$ $S(\tau) S(\sigma)$ whenever $\tau / \sigma$ is rational and $\delta\left(\tau+\sigma, e^{(\tau+\sigma) \psi(\cdot)}|f|_{3}\right)$ is finite. Since both sides of this equation depend continuously on $\tau$, we may drop the requirement that $\tau / \sigma$ be rational. Thus $S$ is a semiflow.

Now from Proposition 3.9 it follows that $A$ is a generator of type $(\alpha, \psi, \omega)$, and that $S(t) \equiv e^{t A}$. This completes the proof of Theorem 5.1.

5.9. Proposition. Let $D$ and $T(t)(t \geqslant 0)$ be continuous linear operators in $a$ Banach space $(E,||)$. Suppose that $\|D\| \leqslant \omega$ and $\|T(t)\| \leqslant e^{t \omega}$ for some constant $\omega \in \mathbf{R}$. Also suppose $\left\|t^{-\beta-1}\left[T(t)-e^{t D}\right]-Q\right\| \rightarrow 0$ as $t \downarrow 0$, for some constant $\beta>0$ and some continuous linear operator $Q$ different from 0 . Then

$$
T\left(\frac{t}{n}\right)^{n}-e^{t D}=O\left(t^{\beta+1} / n^{\beta}\right) \text { as } n \rightarrow \infty,
$$

uniformly for bounded $t$. This rate cannot be improved if $\|Q\|>0$. In fact, a limit

$$
P(t)=\lim _{n \rightarrow \infty} \frac{n^{\beta}}{t^{\beta+1}}\left[T\left(\frac{t}{n}\right)^{n}-e^{t D}\right]
$$

exists uniformly for bounded $t$, and the remainder term $P(t)$ is nonzero for all $t>0$ sufficiently small.

Proof. Define

$$
\varepsilon(t) \equiv \sup _{0<s \leqslant t}\left\|\frac{T(s)-e^{s D}}{s^{\beta+1}}-Q\right\|, \quad P(t) \equiv \frac{1}{t} \int_{0}^{t} e^{(t-s) D} Q e^{s D} d s .
$$

We shall show that the latter function has the properties stated in the proposition. Observe that

$$
\left\|e^{t D}-I\right\|=\left\|\sum_{j=1}^{\infty} \frac{(t D)^{j}}{j !}\right\| \leqslant \sum_{j=1}^{\infty} \frac{(t \omega)^{j}}{j !}=e^{t \omega}-1
$$

hence

Therefore

$$
\begin{aligned}
\left\|Q-e^{\tau D} Q e^{\sigma D}\right\| & \leqslant\left\|Q-e^{\tau D} Q\right\|+\left\|e^{\tau D} Q-e^{\tau D} Q e^{\sigma D}\right\| \\
& \leqslant\|Q\|\left[\left(e^{\tau \omega}-1\right)+e^{\tau \omega}\left(e^{\sigma \omega}-1\right)\right]=\|Q\|\left[e^{(\tau+\sigma) \omega}-1\right] .
\end{aligned}
$$

$$
\begin{aligned}
\|Q-P(t)\| & =\left\|\frac{1}{t} \int_{0}^{t}\left[Q-e^{(t-s) D} Q e^{s D}\right] d s\right\| \\
& \leqslant \frac{1}{t} \int_{0}^{t}\left\|Q-e^{(t-s) D} Q e^{s D}\right\| d s \leqslant\|Q\|\left(e^{t \omega}-1\right) .
\end{aligned}
$$

It follows that $\|P(t)\| \geqslant\left(2-e^{t \omega}\right)\|Q\|$, and so $P(t)$ is nonzero for all $t>0$ sufficiently small. 
From (5.11) we obtain $\left\|T(t)-e^{t D}\right\| \leqslant t^{\beta+1}[\varepsilon(t)+\|Q\|]$. Use this to estimate

$$
\begin{aligned}
\left\|T(s)^{k}-e^{k s D}\right\| & =\left\|\sum_{j=1}^{k} T(s)^{k-j}\left[T(s)-e^{s D}\right] e^{(j-1) s D}\right\| \\
& \leqslant \sum_{j=1}^{k}\|T(s)\|^{k-j}\left\|T(s)-e^{s D}\right\| e^{(j-1) s\|D\|} \\
& \leqslant k s^{\beta+1} e^{k s \omega}[\varepsilon(s)+\|Q\|] .
\end{aligned}
$$

Temporarily fix some positive integer $n$. For $j=1,2,3, \ldots, n$, let

$$
\begin{aligned}
C_{j}= & \frac{1}{n} \exp \left(\frac{n-j}{n} t D\right) Q \exp \left(\frac{j-1}{n} t D\right)-\frac{1}{t} \int_{(j-1) \frac{t}{n}}^{j \frac{t}{n}} e^{(t-s) D} Q e^{s D} d s \\
= & \frac{1}{t} \int_{(j-1) \frac{t}{n}}^{j \frac{t}{n}} \exp \left(\frac{n-j}{n} t D\right) \\
& \cdot\left\{Q-\exp \left[\left(\frac{j}{n} t-s\right) D\right] Q \exp \left[\left(s-\frac{j-1}{n} t\right) D\right]\right\} \exp \left(\frac{j-1}{n} t D\right) d s .
\end{aligned}
$$

Using (5.12), we estimate $\left\|C_{j}\right\| \leqslant n^{-1} e^{t \omega}\|Q\|\left(e^{t \omega / n}-1\right)$. Now compute

$$
\begin{aligned}
& \left\|\frac{n^{\beta}}{t^{\beta+1}}\left[T\left(\frac{t}{n}\right)^{n}-e^{t D}\right]-P(t)\right\| \\
& =\| \frac{n^{\beta}}{t^{\beta+1}} \sum_{j=1}^{n} T\left(\frac{t}{n}\right)^{n-j}\left[T\left(\frac{t}{n}\right)-\exp \left(\frac{t}{n} D\right)\right] \exp \left(\frac{j-1}{n} t D\right) \\
& =\| \frac{n^{\beta}}{t^{\beta+1}} \sum_{j=1}^{n}\left\{T\left(\frac{t}{n}\right)^{n-j}\left[T\left(\frac{t}{n}\right)-\exp \left(\frac{t}{n} D\right)\right] \exp \left(\frac{j-1}{n} t D\right)\right. \\
& \leqslant \frac{n^{\beta}}{t^{\beta+1}} \sum_{j=1}^{n} \| T\left(\frac{t}{n}\right)^{n-j}\left[T \left(\frac{t}{n}{ }^{e^{(t-s) D}} Q e^{s D} d s \|\right.\right. \\
& \quad+\frac{1}{n} \sum_{j=1}^{n}\left\|\left[T\left(\frac{t}{n}\right)^{n-j}-\exp \left(\frac{n-j}{n} t D\right)\right] Q \exp \left(\frac{j-1}{n} t D\right)\right\|+\sum_{j=1}^{n}\left\|C_{j}\right\| \\
& \leqslant \frac{n^{\beta}}{t^{\beta+1}} \sum_{j=1}^{n}\left(\frac{t}{n}\right)^{\beta+1} e^{t \omega} \varepsilon\left(\frac{t}{n}\right) \\
& \quad+\frac{1}{n} \sum_{j=1}^{n}\left(\frac{t}{n}\right)^{\beta+1} n e^{t \omega}\left[\varepsilon\left(\frac{t}{n}\right)+\|Q\|\right]+\sum_{j=1}^{n} \frac{1}{n} e^{t \omega}\|Q\|\left(e^{t \omega / n}-1\right) \\
& =\left\{\varepsilon\left(\frac{t}{n}\right)\left[1+\frac{t^{\beta+1}}{n^{\beta}}\right]+\|Q\|\left[\frac{t^{\beta+1}}{n^{\beta}}+\left(e^{t \omega}-1\right)\right]\right\} e^{t \omega},
\end{aligned}
$$

which tends to 0 as $n \rightarrow \infty$, uniformly for bounded $t$. This completes the proof of (5.10). 
5.13. Corollary. Let $A, B, C$ be generators of type $(\alpha, \psi, \omega)$. Then all the convergences (0.3)-(0.5) hold uniformly on bounded subsets of $E_{3}$, uniformly for sufficiently small $t$. Moreover, in general the rates of convergence in those formulas cannot be improved.

Proof. Using 3.7 and 4.5 , we find that:

$T_{1}(t) \equiv e^{t A} e^{t B} e^{t C}$ is a scheme for $D \equiv A+B+C$ with a remainder $W$ which is, in general, nonzero;

$T_{2}(t) \equiv \frac{1}{2}\left[e^{t A} e^{t B} e^{t C}+e^{t C} e^{t B} e^{t A}\right]$ is a scheme for $D \equiv A+B+C$ with remainder 0 ;

$T_{3}(t) \equiv \exp \left(\frac{t}{2} A\right) \exp (t B) \exp \left(\frac{t}{2} A\right)$ is a scheme for $A+B$ with remainder 0 .

Hence (0.3)-(0.5) follow from Theorem 5.1.

To show that the rate of convergence in $(0.3)$ cannot be improved in general, we note from (3.5) and 3.8 that

$$
\begin{gathered}
\left|T_{1}(t) f-f-t D(f)-\frac{1}{2} t^{2} D^{\prime}(f) D(f)-\frac{1}{2} t^{2} W(f)\right|_{0} \leqslant t^{3} \gamma\left(t,|f|_{3}\right), \\
\left|e^{t D} f-f-t D(f)-\frac{1}{2} t^{2} D^{\prime}(f) D(f)\right|_{0} \leqslant t^{3} \gamma\left(t,|f|_{3}\right),
\end{gathered}
$$

for some bounding function $\gamma$. Subtracting, we obtain

$$
\left|T_{1}(t) f-e^{t D} f-\frac{1}{2} t^{2} W(f)\right|_{0} \leqslant 2 t^{3} \gamma\left(t,|f|_{3}\right) .
$$

For continuous linear operators, then, Proposition 5.9 is applicable, with $Q=\frac{1}{2} W$ and $\beta=1$.

To show that the convergence rates in (0.4), (0.5) cannot be improved in general, we apply Proposition 5.9 with $\beta=2$, again assuming $A, B, C$ are continuous linear operators. We omit the details.

\section{Continuity, Dissipativeness, and Further Remarks.}

6.1. Proposition. Let $A$ be a generator of type $(\alpha, \psi, \omega)$. Let $\rho>0$. Then the restriction of $A$ to the set $\left\{f \in E_{3}:|f|_{3} \leqslant \rho\right\}$ is Hölder-continuous from the metric of $E_{0}$, into $E_{0}$, with exponent $1 / 2$.

Proof. Define $\gamma$ as in Proposition 3.8. Choose $\tau>0$ small enough so that $\rho_{1} \equiv e^{\tau \psi(\cdot)} \rho$ is finite and $\gamma(\tau, \rho)$ is finite. Then if $|f|_{3},|g|_{3} \leqslant \rho$ and $0<t \leqslant \tau$, we have

$$
\begin{aligned}
\left|e^{t A} f-f-t A f\right|_{0} & \leqslant\left|e^{t A} f-f-t A f\right|_{1} \leqslant t^{2} \gamma(t, \rho), \\
\left|e^{t A} g-g-t A g\right|_{0} & \leqslant\left|e^{t A} g-g-t A g\right|_{1} \leqslant t^{2} \gamma(t, \rho),
\end{aligned}
$$

hence

$$
\begin{aligned}
|A f-A g|_{0} & \leqslant \frac{1}{t}\left(\left|e^{t A} f-e^{t A} g\right|_{0}+|f-g|_{0}+2 t^{2} \gamma(t, \rho)\right) \\
& \leqslant \frac{1}{t}|f-g|_{0}\left(1+e^{t \omega\left(\rho_{1}\right)}\right)+2 t \gamma(t, \rho) .
\end{aligned}
$$

Now let

$$
t \equiv \tau \sqrt{\frac{|f-g|_{0}}{2 \rho}} ; \text { then } t \leqslant \tau \sqrt{\frac{|f|_{3}+|g|_{3}}{2 \rho}} \leqslant \tau
$$


and also $t>0$ if $f \neq g$. Thus we can substitute this value of $t$ into (6.2). This yields

$$
|A f-A g|_{0} \leqslant \sqrt{|f-g|_{0}}\left\{\frac{\sqrt{2 \rho}}{\tau}\left(1+e^{\tau \omega\left(\rho_{1}\right)}\right)+\frac{\tau \sqrt{2}}{\rho} \gamma(\tau, \rho)\right\},
$$

whenever $|f|_{3},|g|_{3} \leqslant \rho$, completing the proof.

6.3. Corollary. Let $\|=\|_{0}$ and $\nu()=\|_{3}$. Let $A$ be $\alpha$-regular, and let $\psi, \omega$ satisfy 2.8(b). Then $A$ is a generator of type $(\alpha, \psi, \omega)$ if and only if $A$ is, in the sense of [15], $a$ $\nu$-generator of type $(\psi, \omega)$.

Proof. This is immediate from the definition of $\nu$-generator; see [15].

6.4. Definition. Let $(E,||)$ be a Banach space. Let $B$ be a mapping from some domain $D(B) \subseteq E$, into $E$. We say $B$ is dissipative in $(E,||)$ if

$$
|(I-\lambda B) f-(I-\lambda B) g| \geqslant|f-g|
$$

for all $f, g \in D(B)$ and all $\lambda>0$. Equivalently, $(I-\lambda B)$ is injective, and the resolvent $(I-\lambda B)^{-1}$ is nonexpansive, for all $\lambda>0$.

Several other, equivalent definitions of dissipative can be found in the literature. See [2] for proofs of some of the equivalences.

6.5. Corollary. Let $A$ be $\alpha$-regular. Let $\psi, \omega$ satisfy 2.8(b). Then $A$ is a generator of type $(\alpha, \psi, \omega)$ if and only if the following three conditions hold:

(a) For each $r \in \mathbf{R}_{+}$, the restriction of $A$ to the set $\left\{f \in E_{3}:|f|_{3} \leqslant r\right\}$ is continuous from the metric of $E_{0}$, into $E_{0}$.

(b) For each $r \in \mathbf{R}_{+}$, the restriction of $A-\omega(r) I$ to the set $\left\{f \in E_{3}:|f|_{3} \leqslant r\right\}$ is dissipative in the Banach space $\left(E_{0}, \|_{0}\right)$.

(c) For each $f \in E_{3}$ there exist sequences $\left\{f_{n}\right\}$ in $E_{3}$ and $\left\{\varepsilon_{n}\right\}$ in $(0,+\infty)$ such that

$$
\varepsilon_{n} \downarrow 0, \quad \frac{\left|f+\varepsilon_{n} A(f)-f_{n}\right|_{0}}{\varepsilon_{n}} \rightarrow 0,
$$

and

$$
\limsup _{n \rightarrow \infty} \frac{\left|f_{n}\right|_{3}-|f|_{3}}{\varepsilon_{n}} \leqslant \psi\left(|f|_{3}\right) .
$$

Proof. See 2.7(c) of [15].

6.6. Further Remarks. Crandall and Liggett [6] showed that if $A$ is an operator in a Banach space $(E,||)$ satisfying $R(I-\lambda A) \geqslant \overline{D(A)}$ for all $\lambda>0$ sufficiently small, and if $A-\omega I$ is dissipative for some constant $\omega \in \mathbf{R}$, then there exists a semigroup $e^{t A}$ defined by $\lim _{n \rightarrow \infty}(I-(t / n) A)^{-n}$. For $f \in D(A)$, their proof gave this convergence rate:

$$
\left|e^{t A} f-\left(I-\frac{t}{n} A\right)^{-n} f\right| \leqslant \frac{t|A f|}{\sqrt{n}} .
$$

A better rate can be achieved if we assume more about $A$. It is easy to show that if $A$ is a continuous linear operator, then

$$
\left\|e^{t A}-(I-t A)^{-1}+\frac{1}{2} t^{2} A^{2}\right\|=O\left(t^{3}\right) \quad \text { as } t \downarrow 0 .
$$


Hence, by Proposition 5.9, we have convergence at the rate

$$
e^{t A}-\left(I-\frac{t}{n} A\right)^{-n}=O\left(\frac{t^{2}}{n}\right),
$$

and no faster. It is also easy to show that

$$
\left\|e^{t A}-\left(I+\frac{t}{2} A\right)\left(I-\frac{t}{2} A\right)^{-1}\right\|=O\left(t^{3}\right) \quad \text { as } t \downarrow 0,
$$

and hence, for continuous linear $A$,

$$
e^{t A}-\left[\left(I+\frac{t}{2 n} A\right)\left(I-\frac{t}{2 n} A\right)^{-1}\right]^{n}=O\left(\frac{t^{3}}{n^{2}}\right) .
$$

Formulas like the last one are investigated for some classes of nonlinear, discontinuous operators in a Hilbert space, in [12].

Mathematics Department

Vanderbilt University

Nashville, Tennessee 37235

1. J. M. BALl, "Finite time blow-up in nonlinear problems," Nonlinear Evolution Equations (M. G. Crandall, ed.), Academic Press, New York, 1978.

2. V. BARBU, Nonlinear Semigroups and Differential Equations in Banach Spaces, Noordhoff, Leyden, 1976.

3. H. BrEzIS \& A. PAZY, "Convergence and approximation of semigroups of nonlinear operators in Banach spaces," J. Funct. Anal., v. 9, 1972, pp. 63-74.

4. P. R. Chernoff, "Product formulas, nonlinear semigroups, and addition of unbounded operators," Mem. Amer. Math. Soc., No. 140, 1974.

5. M. G. Crandall, "An introduction to evolution governed by accretive operators," Dynamical Systems (L. Cesari, J. K. Hale and J. P. LaSalle, eds.), Vol. I, Academic Press, New York, 1976.

6. M. G. Crandall \& T. M. LiggetT, "Generation of semi-groups of nonlinear transformations on general Banach spaces,” Amer. J. Math., v. 93, 1971, pp. 265-298.

7. J. Douglas, JR. \& J. E. GunN, "A general formulation of alternating direction methods. I: Parabolic and hyperbolic problems," Numer. Math., v. 6, 1964, pp. 428-453.

8. N. DuNFORD \& J. T. SCHWARTZ, Linear Operators. I, Wiley, New York, 1957.

9. R. HeRSH \& T. Kato, "High-accuracy stable difference schemes for well-posed initial-value problems," SIAM J. Numer. Anal., v. 16, 1979, pp. 670-682.

10. E. Hille \& R. S. Philuips, Functional Analysis and Semigroups, Amer. Math. Soc. Colloq. Publ., vol. 31, Amer. Math. Soc., Providence, R. I., 1957.

11. P. D. LAX \& R. D. RichtmyeR, "Survey of the stability of linear finite difference equations," Comm. Pure Appl. Math, v. 9, 1956, pp. 267-293.

12. P. L. LIONS \& B. Mercier, "Splitting algorithms for the sum of two nonlinear operators," SIAM J. Numer. Anal., v. 16, 1979, pp. 964-979.

13. E. E. Rosinger, "Stability and convergence for nonlinear difference schemes are equivalent, J. Inst. Math. Appl., v. 26, 1980, pp. 143-149.

14. E. SCHECHTER, "Stability conditions for nonlinear products and semigroups," Pacific J. Math., v. 85, 1979, pp. 179-200.

15. E. SCHECHTER, "Interpolation of nonlinear partial differential operators and generation of differentiable evolutions," J. Differential Equations, v. 46, 1982, pp. 78-102.

16. E. SCHECHTER, "Necessary and sufficient conditions for convergence of temporally irregular evolutions," Nonlinear Anal. Theory Methods Appl. (To appear.)

17. G. A. SoD, "A survey of several finite difference methods for systems of nonlinear hyperbolic conservation laws," J. Comput. Phys., v. 27, 1978, pp. 1-31.

18. G. Strang, "On the construction and comparison of difference schemes," SIAM J. Numer. Anal., v. 5,1968 , pp. 506-517.

19. K. YosidA, Functional Analysis, first and later eds., Springer-Verlag, New York, 1964 and later. 